Cambouis, la revue des sciences sociales aux mains sales

\title{
Résistance à la statistique, résistance à la sociologie
}

\section{François Héran}

\author{
INED-INSEE
}

Résumé : L'objectivation du monde social, tâche essentielle de la sociologie, passe par la réduction statistique. C'est en appréhendant « à vol d'oiseau » les trajectoires personnelles qu'on a le plus de chances de les mettre en parallèle et d'en révéler la vérité profonde. Or rien n'est plus malaisé qu'une telle rupture. Rien n'est plus coûteux que cette banalisation de l'irréductible qu'implique l'analyse sociologique du monde social. On se propose de montrer ici que la pratique sociologique ne se définit pas sans la pratique statistique, et réciproquement. Elles se constituent en quelque sorte par une série de prises de position de l'une sur l'autre. On avancera d'abord que la résistance à l'objectivation statistique exprime très couramment une opposition à toute objectivation de type sociologique. Mais cette première proposition sera corrigée par une seconde, à savoir que la statistique peut à son tour constituer le moyen d'échapper aux exigences de l'analyse sociologique. À cela une explication simple: la statistique est objectivement une technique de rupture avec les représentations spontanées, elle n'en est pas encore une théorie. C'est à la sociologie de prendre le relais en tentant d'analyser la distance qui sépare les régularités statistiques des représentations spontanées. Quelques exemples illustreront ce double mouvement et réussiront peut-être à montrer qu'il ne renferme aucune contradiction...

Date de publication : 29/06/2021

Dossier : Sociologie et statistique

Comment citer : $10.52983 /$ crev.vi0.57

Licence:Cambouis publie ses contenus selon les termes de la Licence Creative Commons Attribution - Pas d'Utilisation Commerciale - Pas de Modification 4.0 International. Les auteurices gardent leurs droits de propriété intellectuelle pleine et entière sur leurs articles. 


\section{Résistance à la} statistique, résistance à la sociologie

\section{François Héran INED-INSEE}

«Le cas qui s’était présenté pour moi d'être admis dans la société des Guermantes m'avait paru quelque chose d'exceptionnel. Mais si je sortais de moi et du milieu qui m'entourait immédiatement, je voyais que ce phénomène social n'était pas aussi isolé qu'il m'avait paru d'abord... Sans doute, les circonstances ayant toujours quelque chose de particulier et les caractères d'individuel, c'était d'une façon toute différente que Legrandin

(par l'étrange mariage de son neveu) à son tour avait pénétré dans ce milieu, que la fille d'Odette s'y était apparentée, que Swann lui-même, et moi enfin y étions venus. Mais à vol d'oiseau, comme fait le statisticien qui néglige les raisons sentimentales ou les imprudences évitables qui ont conduit telle personne à la mort, et compte seulement le nombre de personnes qui meurent par an, on voyait que plusieurs personnes parties d'un même milieu, dont la peinture a occupé le début de ce récit, étaient parvenues dans un autre tout différent, et il est probable que, comme il

se fait par an à Paris un nombre moyen de mariages, tout autre milieu bourgeois cultivé et riche eût fourni une proportion à peu près égale de gens comme Swann, comme Legrandin, comme moi et comme Bloch... » Marcel Proust, À la recherche du temps perdu. Le temps retrouvé

Il peut paraître curieux d'ouvrir une réflexion sur les résistances à la statistique et à la sociologie par un texte qui témoigne précisément de l'esprit contraire. Mais cette page, une des dernières écrites par Marcel Proust, montre s'il en était besoin qu'il peut y avoir chez un non-sociologue bien plus de sociologie que chez certains « sociologues ». Elle rappelle dans le même temps que l'objectivation du monde social, tâche essentielle de la sociologie, passe par la réduction statistique. C'est en appréhendant « à vol d'oiseau » les trajectoires personnelles qu'on a le plus de chances de les mettre en parallèle et d'en révéler la vérité profonde.

Marcel Proust l'avait compris, on ne saisit bien son identité sociale qu'en abandonnant le sentiment illusoire d'une identité tout individuelle et autonome, ou, pour reprendre sa formule, en « sortant de soi ». Or rien n'est plus malaisé qu'une telle rupture. Rien n'est plus coûteux que cette banalisation de l'irréductible qu'implique l'analyse sociologique du monde social. Émile Durkheim a souvent noté que la résistance à la sociologie n'a rien qui doive surprendre le sociologue : « quand, comme condition d'initiation préalable, on demande aux gens de se défaire des concepts qu'ils ont l'habitude d'appliquer à un ordre de choses, pour repenser celles-ci à nouveaux frais, on ne peut s'attendre à recruter une nombreuse clientèle »(Durkheim, 1895, dernière page).

De fait, la lutte pour faire admettre la légitimité d'une méthode scientifique d'analyse des faits sociaux, telle qu'Émile Durkheim a contribué à la définir, est loin d'être close. On trouvera peut-être difficile de parler des rapports entre sociologie et statistique si la définition de la démarche sociologique ne peut être considérée comme acquise par tous ceux qui s'en réclament. Comment 
peut-on cerner une relation entre des termes dont l'un donne encore lieu à des luttes de définition?

Il n'est pas sûr en réalité que la question doive se poser ainsi. C'est peut-être dans la relation qu'ils entretiennent que les deux termes, sociologie et statistique, trouvent précisément leur définition, et non l'inverse. On se propose de montrer ici que la pratique sociologique ne se définit pas sans la pratique statistique, et réciproquement. Elles se constituent en quelque sorte par une série de prises de position de l'une sur l'autre. Pour des commodités d'exposition, on distinguera dans ce processus plusieurs étapes ${ }^{1}$.

On avancera d'abord que la résistance à l'objectivation statistique exprime très couramment une opposition à toute objectivation de type sociologique. Mais cette première proposition sera corrigée par une seconde, à savoir que la statistique peut à son tour constituer le moyen d'échapper aux exigences de l'analyse sociologique. À cela une explication simple : la statistique est objectivement une technique de rupture avec les représentations spontanées, elle n'en est pas encore une théorie. C'est à la sociologie de prendre le relais en tentant d'analyser la distance qui sépare les régularités statistiques des représentations spontanées. Quelques exemples illustreront ce double mouvement et réussiront peut-être à montrer qu'il ne renferme aucune contradiction...
1 Le texte qui suit doit beaucoup aux travaux de Pierre Bourdieu, notamment Le sens pratique (Bourdieu, 1980). J'ai tiré profit sur certains points de mes conversations avec Alain Desrosières et Yves Lemel (Insee). Les observations d'Yves Charbit et Benoît Riandey (Ined) ont permis de corriger et de clarifier une première version. Le point de vue exprimé reflète celui d'un sociologue ayant passé par l'ethnologie et travaillant actuellement pour un institut de démographie et, provisoirement, un institut de statistique. Les aspects institutionnels du problème traité seront toutefois omis (voir à ce sujet les communications d'Alain Desrosières à ce colloque). Je précise qu'il ne sera pas question ici de la statistique mathématique, mais de la pratique statistique courante (définition, collecte, analyse de données).

\section{La résistance à la statistique : une forme de résistance à la sociologie}

La statistique est pour le sociologue l'instrument privilégié de la rupture avec les perceptions spontanées. C'est pourquoi l'opposition à la statistique est une forme de résistance à la sociologie. Il y a en effet dans la pratique statistique une manière abrupte, à la fois réductrice et abstraite, de saisir les réalités sociales. Les totalités immédiates n'existent plus pour elle : elle fait fi de l'individu, dont la perception concrète est un des plus grands obstacles épistémologiques que puisse rencontrer la sociologie. Citant le cas des taux de natalité, de nuptialité et le « taux des suicides », Émile Durkheim souligne dès le début des Règles de la méthode sociologique que la statistique est un « artifice de méthode » indispensable, qui offre le moyen d'« isoler » le fait social, c'est-à-dire de le dégager de ses répercussions individuelles. Marcel Proust, on l'a vu, ne disait pas autre chose. Pour le statisticien, les individus ne sont que les supports comptables d'un certain nombre de caractéristiques extérieures, interchangeables avec celles de milliers d'autres individus.

«Ne jamais prendre pour objet de recherches qu'un groupe de phénomènes préalablement définis par certains caractères extérieurs qui leur sont communs et comprendre dans la même recherche tous ceux qui répondent à cette définition $»:$ la statistique ne fait pas autre chose, c'est-à-dire qu'elle suit à la lettre la première des « règles de la méthode sociologique ». Certes, le choix des caractères extérieurs opéré dans la pratique statistique courante (tranche d'âge, tranche de revenus, niveau de diplôme, distinction actifs/ inactifs ou salariés/indépendants, fréquence annuelle d'une pratique culturelle, etc.) répond dans bien des cas à un impératif d'origine administrative ou technique, aisément critiquable par le sociologue. Des découpages aussi courants que les limites d'âge (majorité légale, âge du passage à la retraite, âge des enfants donnant droit à certaines prestations, etc.) sont des enjeux politiques définis par des luttes et des négociations sociales. Alain Desrosières et Laurent Thévenot (1979) ont révélé que les classifications professionnelles, notamment dans les nouveaux métiers de la classe moyenne, ne faisaient pas l'objet d'un consensus général. Tout statisticien soucieux de sociologie ou, tout simplement, de rigueur technique (Volle, 1980) accorde une place 
essentielle à l'élaboration critique des nomenclatures. Mais il ne faudrait pas arguer de cette difficulté évidente pour récuser comme illégitime l'idée même d'une objectivation statistique. Au stade encore très sommaire où l'on se place ici, il faut rappeler que le fait de compter et de chiffrer introduit déjà une première distance dans l'appréhension des réalités sociales. Il ne s'agit pas ici de prôner une sociologie « quantitativiste », comme on le verra plus loin. Aux yeux d'Émile Durkheim lui-même, la première règle de la méthode n'avait précisément qu'une valeur de méthode et non de théorie : la définition extérieure de l'objet « fournit seulement le premier point d'appui nécessaire à une explication » (Durkheim, 1895, p. 42). Mais cette étape est indispensable et la statistique la réalise avec une remarquable efficacité même si c'est souvent en toute innocence. Ainsi, un démographe ${ }^{2}$ aurait beau être personnellement convaincu, par exemple, du caractère purement psychologique des comportements collectifs, il lui est impossible de traduire ce genre de convictions dans sa pratique, et ce en raison des outils dont il dispose : état et mouvement de la population, calcul des taux de natalité, fécondité, nuptialité, etc. C'est que la statistique ne fait pas de sentiment. Et cette indifférence lui confère déjà une valeur sociologique.

D'où l'intérêt qu'offre la statistique dans tous les domaines où le discours des agents se présente apparemment comme l'unique vérité des pratiques, qu'il s'agisse de la religion, de la culture, de la politique, de l'éducation ou de tous les comportements qui semblent résulter d'une « décision » individuelle, tels que le mariage, la fécondité, le choix de la profession, le choix des amis, les cooptations en tout genre, le vote, l'adoption d'une pratique sportive, l'achat d'un vêtement, les goûts alimentaires ou culturels, les choix budgétaires des familles ou le choix d'une méthode contraceptive...

Traiter tous ces champs de pratiques « comme des choses », les faire apparaître dans des colonnes de statistiques, c'est d'abord mettre au jour des régularités systématiques souvent inattendues et qui, selon le mot d'Émile Durkheim, ne sont pas « naturellement compénétrables à l'intelligence». Ce faisant, le sociologue est contraint de s'intéresser aux relations observables entre des variables en apparence éloignées. Mais, non contente de rompre ainsi avec l'intuition commune de l'action, la statistique en prend résolument le contre-pied : c'est de l'extérieur qu'elle prétend procurer une meilleure compréhension des pratiques, alors que leur agent est intimement persuadé d'en être l'acteur, voire l'auteur, et d'en détenir mieux que tout autre une compréhension immédiate. Mettre ainsi à distance la connaissance indigène de l'action, c'est mettre en question la philosophie sociale du « Sujet agissant », une des plus tenaces, une des plus insidieuses des résistances à l'objectivation sociologique. On en retrouve de multiples avatars dans la littérature dite sociologique, sous forme de théories sacralisant le sujet social comme « acteur », « décideur », « individu intentionnel », « joueur», « producteur de la société », « déclencheur de dynamiques sociales nouvelles », etc. La statistique a l'immense mérite de récuser de fait et d'emblée ces variantes de la sociologie spontanée qui consistent toutes à expliquer l'action par une sorte de vis activa à la fois individuelle et générique, ce qui est l'explication anti-sociologique par excellence.

\section{La résistance à la statistique : une opposi- tion à l'atomisation du social ?}

On verra peut-être dans les propos qui précèdent un « éloge » excessif de la statistique. N'a-t-elle pas plutôt pour résultat d'atomiser la société en la réduisant à ces particules élémentaires et interchangeables que sont les individus ?
2 On considérera ici que le statisticien et le démographe se situent sur le même plan, bien que les deux disciplines n'aient pas des origines tout à fait communes et que l'analyse démographique ait développé des méthodes originales (par exemple, la distinction entre transversal et longitudinal, aujourd'hui reprise par les statisticiens). 
La découverte du two-step flow dans la diffusion de l'information, la mise en évidence par Lazarsfeld du rôle des leaders dans ce processus, conduisent à penser que les individus n'ont pas tous le même poids. La statistique présupposerait ainsi l'existence entre eux d'une égalité purement fictive, conforme à l'idéologie libérale. Lui résister serait donc faire preuve de vigilance épistémologique.

Objection capitale, mais dont il faut cerner exactement la portée : c'est à la conception courante des enquêtes d'opinion qu'elle s'applique. Elle vise tout particulièrement le calcul d'une opinion moyenne ou d'une opinion modale. Des commentaires de la forme «le public pense que... », «l'opinion désapprouve... », etc. sont justiciables d'une telle critique. On comprend qu'ils visent si souvent à conseiller le Prince, lequel est par fonction plus intéressé au simple total des voix qu'à leurs différences internes. Le sociologue, pour sa part, décèle dans ce style de formules la résurgence du Sujet sous une forme collective. On peut dire que le développement des sondages d'opinion a permis, de façon inespérée, le retour en force d'une philosophie subjectiviste dans la science sociale, et ce avec d'autant plus de succès qu'elle semble s'abriter derrière la caution objective des chiffres.

Mais, faut-il le rappeler, il ne s'agit là que d'une application seconde et terriblement sommaire de la statistique, généralement réduite à une série de tris à plat, comme en témoignent journellement les publications de sondages dans la presse. Il en va tout autrement lorsque le chercheur prend la statistique au sérieux : il s'engage alors dans l'exploration systématique des différences qui séparent les groupes, relie les opinions aux caractéristiques détaillées des agents qui les professent et analyse les relations entre variables. Le travail de Grunberg et Mouriaux (1979) sur les opinions politiques des cadres est ici exemplaire : on y voit un bloc social couramment présenté comme homogène - les cadres - se dissocier peu à peu, sous les effets de l'analyse statistique, en plusieurs groupes très différenciés. La détection des effets de structure joue dans cette opération un rôle essentiel. Un lien est ainsi établi entre des classes d'opinion et des types de trajectoires sociales. Il est clair que le recours à la statistique n'implique pas en soi une atomisation du monde social. Il constitue au contraire un instrument irremplaçable pour détecter les vraies différences ou les inégalités et repérer des classes de positions et de trajectoires, seul moyen d'identifier autrement qu'en théorie des positions et des trajectoires de classe.

Le fait que les unités statistiques interrogées soient habituellement des individus ne condamne donc pas la statistique à faire de l'individualisme. Nombre de travaux quantitatifs interrogent d'ailleurs une unité plus complexe, le ménage, ensemble d'individus formant indéniablement une unité économique et sociale. Les ménages à leur tour sont classés au moyen de la statistique, à l'instar des individus. Ces procédures permettent, dans l'un et l'autre cas, de cerner précisément ce par quoi les unités considérées se rapprochent ou se séparent. Il en est de même lorsque les unités interrogées sont des entreprises. Rien ne permet de soupçonner dans ces pratiques statistiques une quelconque croyance en l'homogénéité du tissu social. Tout au contraire, elles en révèlent les discontinuités ou les concentrations (au sens de Gini...). Il serait tout à fait inexact d'attribuer à la statistique une sorte d'impuissance congénitale à saisir les rapports entre unités statistiques, qu'il s'agisse des individus, des ménages ou des entreprises. Critique-t-on l'analyse factorielle parce qu'il lui arrive fréquemment de projeter sur ses plans des individus (souvent parfaitement individualisables, alors que la statistique classique les fait vite disparaître) ? La qualité sociologique d'une technique d'objectivation ne dépend pas du matériau de base, en l'occurrence les points individuels, mais du traitement formel qu'on leur applique : ce sont les nuages de points que l'on observe. 
On devrait d'ailleurs s'interroger sérieusement sur l'apport réel de la théorie du leadership à la sociologie. Sa contribution à une critique des propriétés atomisantes de l'enquête d'opinion est indéniable. Mais elle a aussi dangereusement favorisé la thématique confuse de l'«influence personnelle », qui fascine tant les psychosociologues. On retrouve ici un des héritages les plus encombrants de la pensée wébérienne, que la (psycho)sociologie libérale s'est empressée de capter à son profit. Charisme, leadership, influence, autorité personnelle, sens inné du commandement... la dérive est révélatrice : on est passé sans peine du vocabulaire théologique au vocabulaire du «management». Entre les sectes protestantes et les « petits groupes » de la sociométrie d'entreprise, il n'y a peut-être qu'une différence de degré.

La mesure sociométrique des « influences » au sein des groupes restreints semble l'exemple achevé d'une pratique sociale qui doit son succès au fait qu'elle ne rompt pas avec l'individualisme, tout en offrant à frais réduits un ersatz de statistique doublé d'un ersatz de sociologie. Les caractéristiques sociales des membres du groupe y sont presque systématiquement laissées dans l'ombre ou retraduites dans des typologies psychologiques qui les rendent méconnaissables. L'inégalité sociale n'y est plus qu'une « asymétrie », la domination s'y résout en « influence ». Le mot d'ordre devient « cherchez le leader » : on connaît l'exemple, devenu presque canonique ${ }^{3}$, du vendeur de maïs hybride ou de tracteurs qui découvre grâce à la théorie du leadership que le bon moyen d'inonder un village de ses produits est d'ignorer le paysan «lambda » et de s'adresser directement aux notables « influents ». La différence est grande entre cette pondération de marketing et l'attitude du statisticien qui, à l'inverse, doit considérer a priori qu'une unité statistique, individu ou ménage, en vaut une autre et doit être prise en compte au même titre. C'est a posteriori seulement, une fois mis à jour les écarts significatifs, qu'il est en mesure d'apprécier l'inégalité des participations individuelles aux diverses pratiques sociales. D'un côté, donc, le problème est de savoir viser l'élite pour être sûr d'atteindre la masse. De l'autre, il s'agit de prendre en considération toute la population sans distinction.

Entre ces deux voies, la sociologie peut-elle se situer? L'hésitation n'est pas de mise : ce n'est pas contre la statistique, mais avec son aide qu'elle peut contrer les éventuels effets d'atomisation. L'organisation des questionnaires d'enquête et des fichiers de données peut être conçue de manière à relier systématiquement les unités statistiques considérées et celles qui lui sont proches. Ainsi, au lieu d'interroger soit l'homme, soit la femme, un questionnaire peut s'adresser aux deux conjoints successivement. Au lieu d'être centrée soit sur le ménage, soit sur un seul individu du ménage, une étude peut s'articuler en plusieurs questionnaires et tenter de saisir ainsi toute la structure interne du ménage. De même, une observation statistique des entreprises peut consister à questionner parallèlement les divers agents qui les composent. La recherche systématique d'informations sur la génération antérieure (origines sociales) est aussi une manière de prendre en compte les liens de dépendance d'une unité à une autre. On ne développera pas ici ces questions.

\section{Résistance au chiffre et défense du sacré}

Non seulement la statistique ne prend pas l'individu comme atome logique de l'action, mais on peut dire qu'elle va jusqu'à le désacraliser. Sur ce point, Marcel Mauss est peut-être allé plus loin qu'Émile Durkheim dans son jugement sur la statistique. La précieuse propriété qu'elle possède de «traiter les faits sociaux comme des choses » excède pour lui la valeur d'un simple artifice de méthode. Elle n'offre pas seulement le moyen de « dégager les faits sociaux des circonstances individuelles », mais elle implique une appréhension quasi
3 Voir les manuels de Mendras (1975) ou de Boudon (1979). 
matérialiste des faits sociaux. « Au fond, dit Marcel Mauss (1969), tout problème social est un problème statistique. La fréquence du fait, le nombre des individus participants, la répétition au long du temps, l'importance absolue et relative des actes et de leurs effets par rapport au reste de la vie, tout est mesurable et devrait être compté. L'assistance au théâtre ou au jeu, le nombre des éditions d'un livre instruisent sur le prix attaché à une œuvre ou à un sport beaucoup mieux que des pages et des pages de moralistes ou de critiques ». Et il ajoute aussitôt : « la force d'une Église se mesure au nombre et à la richesse de ses temples, au nombre de ses croyants et à la grandeur de leurs sacrifices, et, s'il faut aussi toujours considérer les impondérables en elle, ne considérer que la foi et la théologie est une non moins grave erreur que de les oublier ».

Objectiver le sacré à travers la statistique de ses œuvres, ce n'est pas simplement le mettre à distance, c'est aussi le désacraliser. Opération sciemment « réductrice », sans laquelle il n'est pas de sociologie du religieux, ni d'ailleurs de sociologie tout court. On trouvera peut-être que le texte de Marcel Mauss reste encore en deçà de l'aplomb théorique avec lequel Max Weber, peu de temps auparavant, caractérisait la religion comme un champ de luttes pour la maîtrise légitime du marché des biens de salut. On dira également que ce ne fut pas la statistique qui permit à Max Weber d'en arriver là. En fait, c'est l'immense réservoir de l'histoire et de la géographie des religions exploré systématiquement, qui lui tenait lieu de statistique, parce que s'y dévoilait une série de variations permettant de dégager certains traits invariants, ceux-ci étant à leur tour rapprochés des invariants décelables dans les comportements économiques : concurrence entre les agents, rareté des biens, tendance au monopole, etc. Cette appréhension quasi matérialiste des religions s'appuie sur une opération d'abstraction évidemment plus complexe que l'abstraction de type statistique. Mais elle est aussi moins efficace, moins économe, et finalement plus sujette à caution. On peut penser que s'il avait disposé d'une technique d'objectivation plus directe, Max Weber aurait évité certains développements subjectivistes ou philosophiques (on pense notamment à son insistance sur la sociologie « compréhensive » ou sur le don inné que serait parfois le « charisme»), développements abondamment repris par la sociologie américaine.

L'appréhension statistique des données constitue sans doute une meilleure initiation à la science sociale. Elle conduit à moindres frais au seuil d'une réflexion sociologique, même si cette dernière peut avoir légitimement d'autres origines. Il suffit de voir les résistances que suscite aujourd'hui encore le simple traitement statistique des faits religieux réclamé par les durkheimiens pour comprendre que l'irruption du quantitatif dans le culturel ou dans le sacré n'a rien perdu de son actualité sociologique.

Un exemple révélateur parmi beaucoup d'autres en est l'œuvre de Jean Delumeau, historien au Collège de France, spécialiste du christianisme. « Nous lisons dix fois par an dans toutes sortes de publications, écrit-il, les statistiques d'où l'on conclut hâtivement au dépérissement du christianisme ». Se refusant à penser qu'il s'agisse là d'une remise en cause du christianisme, il préfère mettre en cause la statistique : « nous sommes victimes des chiffres, du quantitatif. Le qualitatif — la sainteté — nous échappe » (Delumeau, 1977). Et plus loin cette prophétie : « le christianisme de l'avenir ne sera pas quantité, mais qualité ». Le chercheur est alors mis en garde contre les «multiples pièges » que comporte l'étude des comportements religieux : « s'appuyant souvent sur des comptabilisations qui portent sur la pratique dominicale, les vocations sacerdotales, les confréries, les retables, etc., l'enquêteur aura-t-il la prétention de calculer la foi ? » Dieu soit loué, Jean Delumeau a trouvé des sociologues qui ont renoncé à un tel sacrilège. Serge Bonnet, par exemple, « sociologue de talent » auteur d'une étude sur les cahiers de prière remplis 
par les fidèles dans certaines études, a eu « la sagesse de ne pas quantifier, et de ne pas ventiler entre d'ennuyeuses et prétentieuses colonnes »...

Il est rare que parmi les chercheurs en science sociale la répulsion vis-àvis de la statistique s'explicite avec une telle candeur. Parce que le public visé débordait le petit monde des pairs, l'auteur ne s'est pas encombré de toutes les précautions oratoires habituellement exigées par la bienséance universitaire. Mais rien n'est plus courant que cette théologie sociale de la statistique, dans laquelle le qualitatif est au quantitatif à peu près ce que l'âme est au corps, et ce que l'élite est à la masse. En l'occurrence, une fois levé le douloureux obstacle de la statistique, l'étude objective des faits religieux glisse sans peine vers un discours religieux sur la religion, qui n'a rien de sociologique, si ce n'est qu'il peut constituer à son tour un document de plus pour une future sociologie de la religion... On voit que les formules de Marcel Mauss n'ont rien perdu de leur actualité. Bien qu'elles semblent apparemment dépourvues des ambitions théoriques de la sociologie wébérienne, elles frappent juste parce qu'elles s'appuient sur une des plus efficaces techniques d'objectivation et de « désenchantement » : la statistique.

Mais, faut-il le rappeler, technique n'est pas théorie. Une méthode de rupture, si efficace soit-elle, ne peut tenir lieu d'épistémologie si elle n'est pas elle-même théorisée. « Quantitativiste », la sociologie doit l'être, mais elle ne peut pas, sous peine d'empirisme, n'être que cela. La voie est donc étroite entre la méthode statistique ou démographique (technique sans théorie sociale) et la philosophie sociale (théorie sans technique).

\section{Régularités statistiques et vision enchan- tée de l'action : un écart dont il faut rendre compte}

La théorie sociologique doit en effet aller au-delà d'un simple usage de l'objectivation : il lui faut accomplir la rupture et néanmoins ne pas cesser de prendre en compte ce avec quoi elle a rompu. En d'autres termes, elle a pour double tâche de « désenchanter » la représentation spontanée du monde social et d'analyser la logique sociale de l'« enchantement». C'est finalement une sociologie de la résistance à la sociologie qui s'impose.

Dans un premier temps, en effet, c'est bien à l'encontre de la sociologie spontanée que, statistiques aidant, le sociologue a des chances de mettre au jour les régularités objectives, les différentiations ou les liaisons systématiques, telles que l'intensité de l'homogamie sociale, le lien entre milieu d'origine et réussite scolaire, la courbe en U de la fécondité, etc.

Mais il faut, dans un deuxième temps, considérer la sociologie spontanée comme faisant elle-même partie du monde social. À ce titre, le sens que les agents attribuent à leurs propres pratiques et leur expérience familière des situations sociales doivent être replacés dans l'analyse sociologique (Bourdieu, 1980, livre I). Sans cette sociologie spontanée, les agents sociaux ne pourraient plus faire ce qu'ils font. Le cas du mariage illustre parfaitement ce phénomène.

On sait d'après plusieurs travaux (Girard, 1964 ; Desrosières, 1978 ; Thélot, 1982) que l'homogamie socioprofessionnelle en France, mesurée par les origines sociales des deux conjoints, est très élevée et varie peu dans le temps, alors que l'idéologie courante du mariage est celle du « libre choix » amoureux et que les familles ont renoncé à intervenir activement dans le choix du conjoint. En fait, le choix matrimonial opère statistiquement un remarquable ajustement des intérêts entre les époux. Mais ce phénomène est l'objet d'une dénégation générale, y compris d'ailleurs dans des milieux traditionnellement enclins à subordonner ouvertement le choix des alliances à la préservation du 
patrimoine, comme les agriculteurs, les patrons ou les professions libérales. Phénomène déjà ancien puisqu'en 1959, selon l'enquête d'Alain Girard, 5 \% seulement des personnes interrogées mettaient le « milieu social » en tête des qualités à considérer chez l'autre en vue d'un mariage ; les autres préférant citer la « santé », les « qualités morales » ou les « goûts communs »...

Le point essentiel, ici, c'est que l'homogamie ne se produit pas en dépit des représentations spontanées du mariage, mais tout au contraire grâce à elles. Sans le mariage d'amour, ce qu'il faut bien appeler le mariage d'intérêt serait psychologiquement intenable et sociologiquement impensable. C'est la méconnaissance de l'homogamie statistique qui la rend praticable. La logique de l'alliance matrimoniale opère donc à un double niveau. Une vérité objective d'abord que la statistique révèle mieux que toute autre technique, à savoir l'homogamie sociale, accord réciproque des atouts et des intérêts en présence qui garantit leur reproduction au sein de la famille. Mais aussi une vérité subjective, l'expérience indigène, familière, du choix amoureux, ce qui « fait courir » les agents sociaux et leur permet de présenter leur conduite comme une conduite désintéressée.

On pourrait citer des exemples équivalents dans d'autres domaines. Ainsi l'éducation : lorsque les enseignants notent, classent et orientent leurs élèves, ils ont le sentiment d'émettre sur eux un jugement personnel, global, qui sanctionne leurs « aptitudes ». On n'imagine pas qu'ils puissent accepter de classer sciemment les élèves en fonction de leurs origines sociales. Cette objectivation brutale du lien entre réussite scolaire et milieu familial aurait tout pour scandaliser les enseignants si une quelconque circulaire ministérielle les enjoignait d'y contribuer... et, pourtant, c'est finalement ce qui se passe statistiquement (Girard et Bastide, 1969). Le jugement professoral suit globalement une logique de sélection sociale. Seulement, pour être mise en œuvre, cette vérité objective implique l'existence chez les professeurs d'une logique subjective du classement qui fait qu'en pratique leur jugement social sur les élèves s'exerce sur le mode psychologique ou essentialiste, à travers un classement des « aptitudes » ou des « personnalités ».

Dans cet exemple comme dans le précédent, la révélation statistique était une première étape, accessible seulement au prix d'une rupture totale avec les représentations spontanées. Remarque triviale peut-être (mais certains sondages d'opinion ne sont pas conçus autrement...), il n'était pas question d'interroger la population mariée ou les agents du système d'enseignement sur la perception qu'ils pouvaient avoir respectivement de l'intensité de l'homogamie ou de la sélection sociale à l'école, pas plus qu'il ne viendrait à un démographe l'idée d'en savoir plus sur le rapport de masculinité en questionnant à ce sujet la population concernée...

Mais, dans une deuxième phase, le sens que les agents sociaux donnent à leurs actions est réintégré dans le champ de l'analyse : libre choix amoureux dans le premier exemple; sélection par les aptitudes en toute neutralité dans le second. Sans ces représentations, les agents ne feraient pas ce qu'ils font. S'ils cessaient d'y croire, ils ne réussiraient plus, comme ils le font, à produire en toute bonne foi un classement social qui élimine statistiquement les mauvais conjoints ou les mauvais élèves et retient ceux dont les caractéristiques sociales ont le plus de chances d'assurer ici la persistance de l'homogamie et là celle des inégalités culturelles.

D'où le problème de taille qui se pose à la sociologie : quels sont les mécanismes concrets qui assurent cette réussite ? Comment se fait-il que les classements spontanés de type psychologique aboutissent à un classement social ? La question est générale. Elle déborde les exemples présentés ici. Elle se pose tout aussi bien dans les cas où ce ne sont pas directement des individus qu'il s'agit de classer, mais des opinions, des pratiques culturelles ou, tout simplement, des objets (eux-mêmes classés par autrui) : préférences en matière 
d'alimentation, d'habillement, d'ameublement, de lectures, de musique, de sports, d'opinions morales, de méthodes contraceptives, et ainsi de suite...

Dans tous ces domaines s'observent des régularités statistiques : les taux d'équipement, les taux de pratique, les profils de consommation ne se distribuent pas au hasard selon les divers groupes sociaux, à commencer par les groupes d'âge. Mais une fois opérés ces constats - parfois extrêmement difficiles à établir - la question qui se pose n'est plus d'ordre statistique, elle est directement sociologique : comment se fait-il que les comportements présentent statistiquement ces reliefs sociaux, alors que les agents concernés y voient pour leur part, selon le cas, de la psychologie, de l'esthétique, de la morale, de l'économie... et non la recherche expresse de classements sociaux? Bref, comment s'accordent concrètement l'objectivité statistique des faits sociaux et leur sociologie spontanée?

Dire que l'une se réalise à l'ombre de l'autre, ou que celle-ci est en quelque sorte l'alibi de celle-là, c'est dire vrai sans doute, mais cela n'explique encore rien. L'interrogation, on le voit, rejoint tout à fait celle que soulèvent les phénomènes d'homogamie ou de sélection scolaire.

On écartera d'abord une réponse devenue classique, celle qui consiste à voir dans les agents sociaux les exécutants d'un « appareil idéologique d'État » ou, plus simplement, d'une idéologie imposée par la classe dominante. Les enseignants, par exemple, sous l'effet d'un système extérieur qui les «écrase », sorte de machine infernale au service des exploiteurs, seraient contraints $\mathrm{d}^{\prime}$ « inculquer massivement l'idéologie dominante ${ }^{4}$. Mais si une explication de ce genre a beaucoup servi dans le cas de la sélection sociale à l'école, elle s'avère tout à fait inutilisable pour rendre compte de l'homogamie. Et pour cause : entre les hommes et les femmes qui se choisissent ou s'évitent sur le champ matrimonial, ne s'interpose aucune instance « extérieure » qui ait le pouvoir coercitif d'un appareil d'État, aucun fonctionnaire qui ait reçu pour fonction de veiller au « bon choix » du conjoint. La forme juridique que doit prendre obligatoirement le mariage ne change rien à cela : le choix du conjoint, qui précède la conclusion du mariage, ne fait pour sa part l'objet d'aucune législation. Juridiquement, le marché matrimonial est libre. Les tentatives eugéniques visant à le contrôler n'ont jamais eu qu'un effet très marginal. Et l'on sait par ailleurs combien est limité aujourd'hui le pouvoir d'intervention de la parenté ou des Églises en la matière. Une des conclusions les plus remarquables que l'on pouvait tirer du Choix du conjoint d'Alain Girard était que la disparition de tout dirigisme ou protectionnisme dans la sélection des alliances n'a nullement empêché ces dernières de rester statistiquement homogames.

On voit tout à fait le profit heuristique que l'on peut extraire d'un exemple comme celui de l'homogamie. Il éclaire en retour celui de la sélection scolaire pour suggérer que dans ce domaine pas plus que dans d'autres le ressort des conduites ne réside dans la contrainte extérieure d'un « appareil » ou dans l'imposition d'une croyance, d'une « norme » ou d'un « rôle » qui ferait violence à l'individu. Il n'est pas sûr que l'usage du mot « idéologie » soit plus pertinent. Certes, l'idéologie est généralement conçue comme une intériorisation de la vision dominante du monde social ou comme l'inculcation d'une « représentation du rapport imaginaire de l'individu à la réalité sociale ». En somme, la résistance à l'objectivation sociologique serait d'origine idéologique. Concept commode, trop commode même. Car il ne suffit pas de découvrir que telle ou telle représentation est « idéologique » pour avoir le fin mot de l'affaire. C'est le mode de fonctionnement de l'idéologie, son mécanisme de formation, qu'il faudrait expliquer.

Sur ce sujet, les théoriciens de l'idéologie sont d'une extrême discrétion. Il semble à les entendre que l'idéologie soit un phénomène essentiellement intellectuel (même s'il s'agit parfois de l'intellect des profondeurs, l'inconscient) ou un phénomène quasi psychologique $\mathrm{d}^{\prime}$ « influence » collective s'exerçant sur
4 Voir Althusser (1976, p. 96). Les mots « système », « écraser», « exploiteurs », « contrainte » sont dans le texte. 
l'individu. Pour lever cette sorte de croyance aveugle, il suffirait de lui dessiller les yeux sur la « réalité objective », en l'occurrence l'exploitation de classe. Et si l'idéologie résiste encore à ces révélations (comme c'est presque toujours le cas), l'explication par la « contrainte » ou par le poids des « appareils » vient prendre le relais — quand ce n'est pas l'explication par la « trahison des propres intérêts de classe » (voir le traitement des classes moyennes dans le marxisme). Louis Althusser a bien perçu ce risque d'une vision purement intellectuelle de l'idéologie ; il a avancé l'idée que l'idéologie se matérialisait dans des « rituels », mais faute de voir que le rituel c'est le geste, et que le geste c'est le corps, il s'est contenté d'y voir une création des appareils d'État. Dans ces conditions, l'automatisme de pensée qui consiste à parler d'idéologie devant tout écart observable entre les pratiques et les représentations cesse d'être utile à la sociologie pour lui faire plutôt obstacle. Il s'agit trop souvent d'une fausse objectivation, d'une objectivation spontanée. Elle privilégie la dénonciation intellectuelle d'un aveuglement intellectuel, au détriment d'une explication sociologique du mécanisme social des représentations. Ce mécanisme, on va le voir, n'est pas celui d'une vision imaginaire, mais tout au contraire celui d'une perception très concrète du monde social, qui est d'abord physique et corporelle. Comment fonctionne cette perception?

La sociologie doit chercher une réponse à cette question dans l'histoire sociale de l'individu. Elle doit tenter de comprendre et d'expliquer l'origine sociale de cette aptitude qu'ont les agents de pouvoir classer les autres instantanément, aux moindres frais, en repérant chez eux un faisceau d'indices pertinents, objectivement corrélés avec leur milieu social. C'est une véritable statistique spontanée que la sociologie doit analyser maintenant.

Dans l'exemple du choix amoureux, les indices perçus sont divers : c'est en discernant l'un chez l'autre les goûts, les manières, les habitudes linguistiques, les pratiques culturelles ou sportives et, plus simplement, l'allure ou la tenue du corps que les candidats au mariage se « situent ». Ils repèrent ce faisant le décalage ou l'ajustement de leurs propriétés sociales respectives. Le « coup de foudre » illustre la façon dont s'impose parfois aux individus la découverte d'une coïncidence entre leurs propriétés sociales, ressentie comme une véritable harmonie préétablie, dès lors que l'histoire sociale de leur constitution est suffisamment proche.

Comme le disait Alain Girard, les futurs conjoints ne se cherchent pas, ils se trouvent. Mais, peut-on ajouter, ils ne se trouveraient pas s'ils n'étaient d'abord capables de se situer. Chose d'autant plus aisée que les agents sociaux sont prédisposés à « reconnaître » et à apprécier les caractéristiques sociales de ceux qui ont statistiquement le plus de chances d'avoir vécu les mêmes situations qu'eux. Il est clair que les caractéristiques qui assurent la reconnaissance réciproque dans la vie courante sont rarement celles que la statistique classique a l'habitude de retenir : les futurs conjoints n'ont aucunement besoin d'échanger leurs cartes de visite professionnelles, leurs fiches de paie ou leurs attestations de diplôme pour se situer dans le feu de l'action ; c'est à partir des évidences les plus visibles qu'ils se prononcent. Et le corps joue à ce stade un rôle déterminant.

On pourrait appliquer au corps ce qu'Émile Durkheim disait de la « morphologie ». Sous des apparences naturelles, le morphologique n'est que du physiologique cristallisé, consolidé au cours de l'histoire. Citant les exemples du réseau de communication, de la densité urbaine, des structures démographiques ou de la monnaie, Durkheim évoquait ces « manières de faire » devenues avec le temps des « manières d'être », c'est-à-dire des pratiques à ce point objectifiées que personne ne songe plus à les modifier. Le géographique ou l'économique ne sont pas autre chose que du social naturalisé. C'est peutêtre aux propriétés du corps que ces observations s'appliquent le mieux : sous des dehors naturels, biologiques, la « morphologie » individuelle incorpore 
toute une histoire physiologique. Derrière les «manières d'être » du corps se cachent et se manifestent à la fois les « manières de faire » passées. Par son gabarit, sa force, ses formes, son esthétique, sa raideur ou sa souplesse, sa gêne ou son aisance, sans compter la voix et l'accent... le corps extériorise l'histoire sociale du sujet, c'est-à-dire une succession d'expériences dont la probabilité est extrêmement inégale d'un individu à l'autre, parce qu'elle dépend des origines sociales et des conditions d'existence. Ainsi, le dressage parental de la tenue et de la prononciation, l'expérience précoce du travail physique, l'usage exclusivement sportif ou cosmétique du corps, la nature du régime alimentaire... sont autant de manières de faire ou de subir qui marquent durablement l'apparence physique.

Ces expériences corporelles seraient d'ailleurs en partie repérables à travers des enquêtes de type statistique. Une enquête de consommation révèle des profils sociaux très accusés du point de vue du régime alimentaire (Grignon et Grignon, 1980). Une enquête de budget-temps donne des indications précieuses sur le temps que chacun consacre à la cosmétique et aux soins du corps. Une description systématique des postes de travail est utile pour décrire en détail les contraintes physiques dans les métiers ouvriers; et ainsi de suite. On peut par conséquent définir en termes statistiques des « classes » d'expériences corporelles. Elles correspondent à des conditions d'existence relativement homogènes et elles engendrent à leur tour des marquages corporels durables qui démarquent empiriquement des classes d'individus.

En bref, le corps tend à classer parce que l'on a tendanciellement le corps de sa classe. C'est à tout moment un indicateur sensible de la trajectoire sociale. Il constitue dans l'expérience familière du monde social le support des classements sociaux spontanés, notamment dans les pratiques de sociabilité: choix des amis, choix du conjoint et autres phénomènes de sélection ou de cooptation (que l'on songe par exemple au rôle joué par l'image physique du candidat dans les oraux d'examen...).

On pourrait parler tout autant d'une véritable statistique spontanée ; les individus parient en quelque sorte sur l'existence de corrélations entre certaines caractéristiques visibles (corporelles, certes, mais aussi linguistiques, vestimentaires...) et des propriétés sociales qui le sont moins. L'expression de « statistique spontanée » peut sembler paradoxale dans la mesure où il s'agit d'une statistique sans chiffre et sans calcul. Il est cependant difficile de trouver une expression aussi concise et aussi parlante pour désigner une pratique en vérité quotidienne. Chacun de nous perçoit immédiatement, souvent sans se l'expliciter, si telle expression de vocabulaire, telle tournure de phrase est banale, fréquente, ordinaire, rare ou exceptionnelle. Le discernement entre ce qui est vulgaire, normal ou recherché présuppose une appréciation des fréquences relatives. Tout le monde sait qu'on peut percevoir un risque (risque de subir une agression, risque de décéder avant tel âge, risque de faire plus de « mauvaises rencontres » en deuxième classe qu'en première, etc.) sans rien savoir du calcul des probabilités. Les commentaires de certains enseignants sur la « baisse du niveau » d'une génération d'élèves à une autre sont un cas typique de statistique spontanée. Un autre (signalé par Henri Leridon) est celui qui touche à la perception de la conjoncture démographique («il y a beaucoup de femmes enceintes dans la rue en ce moment »...). La conjoncture économique donne lieu à des flots de statistique spontanée. Il est clair toutefois qu'il est souvent difficile de dire dans quelle mesure elle n'est pas contaminée par une manipulation très peu «spontanée » des informations chiffrées, due à ceux qui détiennent de fait le monopole de leur diffusion auprès du grand public. Dans le registre qui nous occupe ici, la statistique spontanée qui relie les traits physiques aux propriétés sociales est une sorte de pari qui offre l'immense avantage d'économiser à l'individu des investigations parfois coûteuses et malaisées. Mais comme tout pari, cette perception est très 
ambiguë : elle dit deux choses à la fois. D'une part, elle traduit partiellement une réalité objective, d'autre part, elle révèle indirectement la position de celui qui en fait montre. On peut dire qu'elle est à la fois fondée socialement et socialement biaisée.

Fondée, parce qu'elle correspond toujours dans une certaine mesure à une réalité statistique objective. Par exemple, un jugement du type «il est gros et moustachu : ça doit être un petit patron », jugement très courant (tout au moins dans certains milieux) a des chances d'exprimer une corrélation vérifiable. Une enquête de l'INSEE (Charraud et Valdelièvre, 1981) a permis d'établir que la plus grosse silhouette est bien celle des patrons du commerce et de l'artisanat, à égalité avec les agriculteurs. Ils ont, comparés aux autres catégories sociales, une taille moyenne associée au poids le plus élevé. Et il ne serait pas impossible par ailleurs qu'une éventuelle statistique sur le port de la moustache par catégories socioprofessionnelles ne vienne encore conforter ce jugement de reconnaissance spontané... Si elle n'avait, en effet, aucun fondement dans une expérience objective locale, une telle image n'aurait pas actuellement le succès qui est le sien. Ce n'est pas par hasard que les dessinateurs ayant pour cible le « Français moyen » ou le « téléspectateur moyen » choisissent cette silhouette pour l'objectiver. Le caricaturiste ne fait que combler les attentes suscitées par la statistique spontanée ("gros + moustache » corrélé avec « classe moyenne »).

Mais l'opération est aussi socialement biaisée, manipulée, comme en témoigne le succès rencontré auprès des intellectuels et des cadres par le portrait du «beauf » dessiné par Cabu : personnage gros et moustachu, mi-épicier mi-policier, intolérant et raciste, censé incarner les menaces fascisantes que la masse fait peser sur la société et la culture... On passera sur le fait que dans une représentation de ce genre, par laquelle une classe sociale (ou une fraction de classe) en stigmatise une autre, l'intolérance — pour ne pas dire le racisme — n'est pas du côté que l'on croit. Si le plus violent des ethnocentrismes de classe peut s'exprimer ainsi en toute quiétude, c'est qu'il le fait sur le mode physique, c'est-à-dire en deçà du discours, de corps à corps, en tablant sur ce que, plagiant les informaticiens, on pourrait appeler la « reconnaissance automatique des formes ». L'assentiment de la partie du public socialement intéressée à ce démarquage est acquis d'emblée (cas, entre autres, comme le signale Luc Boltanski (1975), des fractions nouvelles des classes moyennes, qui ne veulent rien avoir de commun avec leurs fractions traditionnelles : artisans, commerçants, patrons ou contremaîtres). « C'est tout à fait ça », dira le lecteur, sans nul besoin d'en dire davantage, ce qui lui permet d'économiser des discours explicites tels que « la classe moyenne, voilà l'ennemi », discours trop exposés au risque d'une critique collective. Une caricature, on le sait, est pratiquement inattaquable.

D’une manière générale, le fait de classer empiriquement les gens sur la base des évidences visibles aboutit à ce résultat inespéré qu'une diffusion publique des classements sociaux les moins acceptés commence à devenir acceptable. Les aversions qu'inspirent les tenues, les accents, l'écriture, les goûts, les manières, les prénoms, etc. ne sont qu'une forme euphémisée, légitimée, des répugnances sociales entre les groupes 5 .

Ne donner que le corps à percevoir, c'est donc évoquer le monde social sans en parler. C'est le «situer » sans le nommer, le faire reconnaître sans lever la méconnaissance dont il est l'objet. Le corps, en effet, n'est qu'un indicateur de l'histoire sociale du sujet. Cela ne veut pas dire qu'il suffise de l'examiner comme on consulte un indicateur des chemins de fer pour y trouver à coup sûr le renseignement désiré. Entre le corps et la classe, il y a seulement corrélation, donc liaison partielle et brouillée. Pour la perception familière du monde social, progressivement formée à travers une statistique spontanée des corrélations, les caractéristiques physiques des individus sont une com-
5 La censure quasi totale qui s'exerce aujourd'hui sur les classements racistes ne contredit pas ces observations. Il semble même qu'elle serve d'alibi à tous les autres classements corporels, dont la logique sociale est au fond identique, mais plus discrète. La couleur a été rejetée au profit d'autres critères ni trop voyants (ce qui était le cas) ni trop visibles. 
posante clef, mais une composante seulement, d'un indicateur synthétique plus complexe. Interviennent également des caractéristiques, linguistiques, vestimentaires, alimentaires, culturelles, anthroponymiques, etc. Ces signes ne sont pas déchiffrés un à un, mais tirent leur signification de la structure dont ils font partie : c'est un faisceau d'indices intégrés dans une perception globale, indices inégalement pondérés d'un groupe social à l'autre.

Ainsi le sens de chaque indice peut être spontanément modifié et réinterprété en fonction de la présence d'un autre indice. Une même taille n'est pas perçue de la même façon selon qu'elle s'associe à un poids lourd ou léger. De même, lorsque tout le corps est à son tour combiné à d'autres traits, des correctifs interviennent en cas d'incohérence partielle. Ces phénomènes ont été étudiés dans des régions exotiques du monde et, curieusement, fort peu dans nos sociétés. Ainsi, un dicton haïtien rappelle que « le noir riche est mulâtre » (couleur des métis, plus valorisée) tandis que « le mulâtre pauvre est noir $»^{6}$. De la même manière, on constate dans les Andes qu'un Indien qui cesse de parler aymara ou quechua et de travailler la terre de ses mains se fait classer comme métis, alors qu'inversement le métis ou même le blanc contraint de travailler lui-même sa terre risque d'être reclassé comme indien. Il n'en va pas autrement dans une société comme la nôtre, où telle personne, apparemment déclassée par son « accent » régional, peut se reclasser grâce à des atouts de type économique ou scolaire. Telle autre, au contraire, pourvue des biens les plus rares, sera peut-être «trahie » par sa prononciation ou son rapport au corps, empreint de gêne et de maladresse. La statistique spontanée sait parfaitement opérer ce genre de «pondérations ». Elle peut même tirer de ces pondérations une déduction intuitive de ce que Pierre Bourdieu appelle la « pente » de la trajectoire sociale, reconstituée grâce aux décalages subtils qui désignent les parvenus, les héritiers de vieille souche, les reconvertis ou les déclassés... Marcel Proust a, mieux que tout autre, aiguisé cette faculté générale de discernement en l'appliquant au microcosme des salons parisiens.

On ne prolongera pas ici l'analyse du « sens pratique » qui oriente les conduites individuelles tout en suivant des régularités statistiques objectives. L'exemple de l'homogamie peut s'étendre à toutes les conduites qui consistent à trier « au jugé » non pas des individus, mais des biens, car ces biens tirent une signification sociale du fait qu'ils sont eux-mêmes classés et diversement appropriés par d'autres individus et donc marqués eux aussi. On peut donc avancer d'une manière générale que le dépassement du constat statistique passe par une compréhension sociologique très fine de la «statistique spontanée » mise en œuvre par les agents sociaux. Cette compréhension peut être qualifiée aussi bien d'ethnologique ou d'anthropologique. Mais il ne faut pas oublier toutefois que cette étape de la recherche ne doit pas court-circuiter l'étape de l'approche statistique. Rien n'est plus dangereux que de chercher immédiatement à « comprendre » les actions d'autrui en essayant de se mettre «à sa place ». L'intuition phénoménologique de l'action brûle l'étape cruciale de la mise au jour des régularités statistiques, elle mise spontanément sur le sens intime au détriment des caractères extérieurs, ouvrant ainsi la voie à toutes ces variantes psychologisantes ou économistes de la sociologie que l'on connaît aujourd'hui, notamment dans la sociologie d'origine américaine.

On ne niera pas pour autant que, sous certaines conditions, l'appréhension des faits sociaux « à vol d'oiseau » ou « à distance » puisse ne pas être de type statistique. L'observation systématique des rituels et de leur variation, des mythes et de leurs variantes, est une méthode ethnologique qui peut se substituer avec profit à la méthode statistique. De même, l'étude historique de certains documents réalise les réquisits indispensables de l'objectivation. À ces exemples près, il reste vrai qu'il n'y a pas de bonne sociologie ou même de sociologie tout court sans le passage par la statistique. Et il est non moins vrai que le dépassement de la statistique est indispensable si l'on veut poursuivre
6 Renseignement communiqué par Yves Charbit, qui développe ce thème dans une thèse en cours sur la nuptialité aux Caraïbes. 
la recherche sociologique en vue de procurer une meilleure compréhension de la logique des comportements.

\section{La statistique : un moyen de résistance à la sociologie?}

Faute de s'interroger sur les médiations et les processus concrets qui relient les caractéristiques des agents à leurs pratiques, le statisticien ou le sociologue « quantitativiste » sont tentés de concevoir la société comme un jeu de variables. Cette déviation de la statistique est à son tour une forme particulièrement insidieuse de résistance à la sociologie.

Ainsi, le moyen le plus sûr d'esquiver l'exigence d'une élucidation sociologique de la reproduction sociale entre les générations consiste peut-être à s'attarder sur les tableaux de mobilité sociale, l'instrument finissant par occulter la réalité qu'il était censé objectiver. On n'en finirait pas d'énumérer les auteurs d' « indices de mobilité sociale », tous plus raffinés les uns que les autres, qui omettent de poser sur leurs présupposés de construction les questions les plus élémentaires. C'est contre ce courant dominant que Claude Thélot a orienté son ouvrage récent sur la mobilité sociale (Thélot, 1982), avec la conviction que l'analyse ne pouvait progresser qu'en analysant d'abord son propre instrument.

Les tables de mobilité, rappelle Claude Thélot, sont des « instruments réducteurs ». Cela ne condamne pas toute utilisation qui en serait faite — une table de mobilité a de toute évidence un pouvoir de révélation considérable - mais cela oblige à manier prudemment l'instrument et à savoir à temps l'abandonner pour d'autres quand la recherche d'une explication plus poussée devient nécessaire. En l'occurrence, de telles tables ne permettent de considérer que la position sociale d'un seul des parents ; elles supposent que d'une génération à l'autre la définition des catégories sociales est restée constante ; elles ne font valoir que des immobilités ou des mouvements formels très dépendants du degré de détail des nomenclatures. De plus, Claude Thélot montre sur plusieurs exemples que les tables sous-estiment l'inertie sociale.

Mais la principale critique qu'il émet à l'encontre des tables de mobilité est qu'elles signalent départs et arrivées tout en laissant totalement dans l'ombre les cheminements. Pour tenter de les reconstituer, il étudie successivement quelques étapes clés : la position sociale en début de carrière, la détention d'un diplôme, les aspirations professionnelles des adolescents. Ce qui l'amène à faire justice des modèles de transmission du statut « en cascade», notamment du modèle selon lequel l'effet des origines sociales sur les positions s'exercerait tout entier à travers le diplôme. En réalité, les détenteurs d'un même diplôme peuvent, selon leur origine sociale et, plus précisément, l'ancienneté de la culture familiale, faire varier considérablement le « rendement » social ou économique de ce diplôme. De même, la mobilité en cours de carrière n'est souvent qu'une récupération plus ou moins tardive du statut paternel (phénomène de « contre-mobilité »). Devant ces effets directs de l'origine sociale, véritable « force de rappel », dit Claude Thélot, il faut bien conclure que « l'influence du milieu d'origine se pérennise durant toute la vie active des descendants, indépendamment de leurs études et de leur position initiale ». Proposition illustrée ensuite dans plusieurs domaines (choix du conjoint, fécondité et options politiques), où s'observe systématiquement l'« empreinte de l'ascendance », que l'on pourrait appeler également l'« effet de trajectoire».

Ce qui frappe le sociologue dans ce travail, c'est d'abord la démonstration de la nécessité d'un passage par la statistique pour l'approche sociologique 
d'un phénomène aussi essentiel que celui de la mobilité sociale. Mais c'est aussi, réflexion faite, l'absence d'une explication proprement sociologique du phénomène. Si l'origine sociale est une « force de rappel », qu'est-ce donc que cette force ? Que signifie l'« influence » d'une telle variable dans la pratique sociale courante? Qu'est-ce qui, très concrètement, se transmet d'une génération à l'autre, et comment cela se transmet-il ?

La sociologie, dans cet exemple, se sépare peut-être de la statistique parce qu'elle s'interroge sur la nature précise des atouts transmis d'une génération à l'autre, atouts multiformes qui ne semblent pas se réduire aux indicateurs habituels (profession, revenu, diplôme...), mais englobent également des propriétés para-culturelles ou para-économiques telles que la maîtrise du langage, l'accent, la tenue, la présentation vestimentaire, les goûts, le « sens des relations », le sens du commerce, le sens du placement, et ainsi de suite... Bref, toutes ces «manières de faire » plus ou moins incorporées dont il était question plus haut. De proche en proche, l'interrogation sociologique se porte sur les ressorts possibles de la mobilité sociale, à commencer par l'inégalité sociale elle-même, les concurrences entre groupes, les stratégies de démarquage ou de conformité, de reclassement ou de déclassement... On objectera peut-être qu'il n'appartient pas au statisticien de s'aventurer dans des hypothèses générales. Mais le renoncement à l'analyse sociologique peut conduire le statisticien à défendre inconsciemment une thèse d'une extrême généralité, celle selon laquelle la mobilité sociale serait exclusivement une affaire de régularités statistiques, un jeu de variables sans joueurs et sans enjeu définis. Le rôle du statisticien se bornerait alors à dresser des gardefous contre la tentation des « modèles » simplistes et celle des explications spontanées. Au-delà, l'« interprétation » serait confiée aux sociologues avec tous les risques de déviation que l'on peut craindre...

Accepter une telle division du travail reviendrait à conforter aussi bien les résistances à la sociologie que les résistances à la statistique. Les deux disciplines se verraient ainsi tronquées, alors qu'une approche scientifique du monde social exige d'elles une constante interaction. En réalité, les constats statistiques appelleront toujours des interprétations sociologiques, et celles-ci susciteront, comme elles commencent déjà à le faire, de nouvelles statistiques. 


\section{Bibliographie}

Althusser Louis, 1976, Positions (1964-1975), Paris, Les Éditions sociales. BolTANSKI Luc, 1975, « La constitution du champ de la bande dessinée », Actes de la recherche en sciences sociales, vol. 1, $\mathrm{n}^{\circ} 1, \mathrm{p}$. 37-59.

Boudon Raymond, 1979, La logique du social. Introduction à l'analyse sociologique, Paris, Hachette.

Bourdieu Pierre, 1980, Le sens pratique, Paris, Éditions de Minuit.

Charraud Alain et VAldelièvre Hélène, 1981, « La taille et le poids des Français », Économie et statistique, $\mathrm{n}^{\circ} 132$, p. 23-38.

Delumeau Jean, 1977, Le christianisme va-t-il mourir ? Paris, Hachette.

Desrosières Alain et ThÉvenot Laurent, 1979, « Les mots et les chiffres : les nomenclatures socioprofessionnelles », Économie et statistique, $\mathrm{n}^{\mathrm{o}} 110, \mathrm{p} .49-65$.

Desrosières Alain, 1978, « Marché matrimonial et structure des classes sociales », Actes de la recherche en sciences sociales, vol. 20-21, p. 97107.

DURKHEIM Émile, 1895, Les règles de la méthode sociologique, Paris, PUF.

GIRARD Alain et BASTIDE Henri, 1969, « Orientation et sélection scolaires : cinq années de promotion, de la fin du cycle élémentaire à l'entrée dans le 2e cycle du second degré », Population, vol. 24, n 1, p. 195-261 et vol. 24, $\mathrm{n}^{\circ} 2$, p. 9-46.

GIRARD Alain, 1964, Le choix du conjoint : une enquête psycho-sociologique en France, Paris, PUF/INED.

GrignON Claude et GRIGNON Christiane, 1980, «Styles d'alimentation et goûts populaires », Revue française de sociologie, vol. 21, $\mathrm{n}^{\circ}$ 4, p. 531569.

Grunberg Gérard et Mouriaux René, 1979, L'univers politique et syndical des cadres, Paris, Presses de Sciences Po.

MAuss Marcel, 1969, « Divisions et proportions des divisions de la sociologie (1927) », dans Essais de sociologie, Paris, Minuit, p. 178-245.

Mendras Henri, 1975, Élements de sociologie, 2e édition, Paris, Armand Colin.

THÉLOT Claude, 1982, Tel père tel fils ? Position sociale et origine familiale, Paris, Dunod.

Volle Michel, 1980, Le métier de statisticien, Paris, Hachette. 